\title{
DISKUSSION
}

\section{Die vorzeitige Beendigung der Wahlperiode des Bundestages: Vorrecht des Parlaments oder Recht des Bundeskanzlers? Zum Aufsatz von Stefan Ulrich Pieper in Heft 2/2007 und dem Diskussionsbeitrag von Karlheinz Niclauß in Heft 3/2007 der ZParl}

\author{
Sven Leunig
}

Stefan Ulrich Pieper diskutiert die Frage, ob ein Selbstauflösungsrecht die Position des Bundestages gegenüber dem Recht des Bundeskanzlers stärken kann, eine Parlamentsauflösung über die Vertrauensfrage gemäß Art. 68 GG zu erwirken. Er vertritt die Position, dass dies nicht der Fall und auch gar nicht notwendig sei, da der Bundestag souverän genug sei, eine Auflösung gegen den Willen seiner Mehrheit zu verhindern. Der Kanzler könne den Bundestag nicht zur Auflösung zwingen. ${ }^{1}$ Darüber hinaus sei, so Pieper, die Einführung des Selbstaufösungsrechts auch aus anderen Gründen nicht vertretbar. So liege es etwa als direktdemokratisches Element mit seiner Verkürzung des Wahlrechts des Bürgers „quer zum Repräsentativsystem “2 . Zudem hätten die Verfassungsväter den Bundestag ganz bewusst daran hindern wollen, „sich (...) durch eine vorzeitige Selbstauflösung der Verantwortung zu entziehen“3. Gegen das Selbstauflösungsrecht spräche daher das „Interesse an der Stabilität einer einmal gewählten Volksvertretung“4 .

Den Vorbehalten gegen das Selbstauflösungsrecht für den Bundestag stimmt Karheinz Niclauß in seiner Replik ,nahezu uneingeschränkt "5 zu, schätzt die Position der Bundestagsabgeordneten im Verfahren nach Art. 68 GG aber deutlich schwächer ein als Pieper. So sei die Zustimmung der Opposition zu einer Vertrauensfrage des Kanzlers aus systemlogischen Gründen nicht zu erwarten: Man spreche „eben nicht dem gegnerischen Bundeskanzler das Vertrauen aus"6, selbst wenn man dem Auflösungsbegehren an sich zustimme. Für die Mehrheitsfraktionen stelle sich das Problem aus demselben Grund genau entgegengesetzt dar: Es werfe schon aus wahltaktischen Gründen kein gutes Licht auf eine Regierungspartei, dem eigenen Kanzler das Vertrauen zu verweigern, auch wenn er dies gerade

1 Vgl. Stefan Ulrich Pieper, Das Selbstauflösungsrecht für den Bundestag als Korrektur des Art. 68 GG? Anmerkungen zur Entscheidung des Bundesverfassungsgerichts vom 25. August $2005-2$ BvE 4/05, in: ZParl, 38. Jg. (2007), H. 2, S. 287 ff., S. 289 f.

2 Ebenda, S. 295.

3 Ebenda, S. 294.

4 Ebenda, S. 295.

5 Karlheinz Niclauß, Echte und aufösungsorientierte Vertrauensfrage. Eine Replik auf Stefan Ulrich Pieper in Heft 2/2007 der ZParl, in: ZParl, 38. Jg. (2007), H. 3, S. 667 f., S. 667.

6 Ebenda, S. 667. Dass die oder bei mehreren Parteien eine der Oppositionsfraktion(en) einem solchen Antrag zustimmt, wäre meines Erachtens nur denkbar, wenn sich vor der Antragstellung eine neue Koalition aus der größeren - also den Kanzler stellenden - Fraktion der bisherigen Regierungskoalition und einer der Oppositionsfraktionen gebildet hätte. Die Vertrauensfrage würde dann zur parlamentarischen Bestätigung dieser neuen Koalition dienen. 
wolle. ${ }^{7}$ Somit werde die Bundestagsauflösung nach Art. 68 GG zu einem „Verwirrspiel der falschen Fragen und falschen Antworten" .

Dem ist im Ergebnis zuzustimmen und nur einiges ergänzend hinzuzufügen: Denkbar wäre zwar, dass einzelne, nicht der Kanzlerpartei angehörende Mitglieder einer Koalitionsfraktion entgegen dem erklärten Willen ihrer Fraktionsführung in ausreichender Zahl dem Kanzler das Vertrauen verweigern. Wahrscheinlich ist das aber nicht, da dies - im Falle einer dann zu erwartenden Auflösung des Bundestags und anschließenden Neuwahl - mindestens ihre erneute Nominierung in Frage stellen würde. In jedem Fall würden sie ihrer Partei erheblichen Schaden zufügen, denn deren Wahlchancen hängen ja nicht unwesentlich vom Grad der Geschlossenheit ab, mit dem sie gegenüber den Wählern auftritt. Dies gilt noch verstärkt und aus demselben Grunde für ein verfassungsrechtlich mögliches, aber ebenso unwahrscheinliches ablehnendes Votum durch einzelne Fraktionsmitglieder der Kanzlerpartei. ${ }^{9}$

Ausschlaggebend ist und bleibt zunächst der Wille des Kanzlers: Will er sich tatsächlich des Vertrauens der Abgeordneten versichern oder - was wahrscheinlicher und auch im Sinne der Intention von Art. 68 GG ist - die Abstimmung mit einer Sachfrage verbinden, um die eigenen Abgeordneten zu disziplinieren, wird ihm das Vertrauen aller Wahrscheinlichkeit nach ausgesprochen werden. Strebt er hingegen eine Auflösung an, so werden ihm seine Fraktion und wohl auch der Koalitionspartner das Vertrauen wunschgemäß nicht aussprechen. Zwar betont das Bundesverfassungsgericht, es sei „keine Lage denkbar, in der ein Bundeskanzler das Parlament gegen dessen Willen rechtlich zwingen könnte, an seiner eigenen Auflösung mitzuwirken (Hervorhebung S.L.) "10. Die öffentliche Erklärung, Neuwahlen anzustreben, entfaltet aber eine starke Bindungswirkung gegenüber den Abgeordneten der Regierungsfraktionen, so dass die Einschätzung Piepers, die Abgeordneten seien

7 Dies gilt zumindest für die Abgeordneten der Kanzlerpartei selbst. Die Abgeordneten einer Koalitionsfraktion könnten hingegen schon gewillt sein, dem Kanzler das Vertrauen zu verweigern, wenn deren Partei sich mit dem Gedanken trägt, die Koalition zu verlassen. In einem solchen Fall tatsächlich unsicherer Mehrheiten wird der Kanzler aber vermutlich von dem Verfahren nach Art. 68 nur Gebrauch machen, wenn er die kritische Lage der Koalition erkennt, die Mandatszahl seiner eigenen Partei bei Neuwahlen verbessern zu können glaubt und dies aus der taktisch günstigeren Position des amtierenden Regierungschefs heraus tun will. Dem Koalitionspartner bleibt dann nur die Wahl, entweder tatsächlich einer Auflösung via Vertrauensverweigerung zuzustimmen (und mögliche Stimmenverluste bei den vorgezogenen Neuwahlen hinzunehmen) oder aber den Weg einer neuen Koalitionsbildung mit der Opposition oder Teilen von dieser zu beschreiten. Dann aber würde ohnehin nicht die Vertrauensfrage, sondern das konstruktive Misstrauensvotum nach Art. 68 GG der wahrscheinliche Ausweg aus dieser Situation sein.

8 Karlheinz Niclauß, Echte und auflösungsorientierte Vertrauensfrage, a.a.O., S. 668.

9 Zur Frage der Geschlossenheit als wesentlicher Bedingung politischen Erfolgs siehe Jürgen Dittberner, Freies Mandat und politische Geschlossenheit. Widerspruch oder Ergänzung zweier Prinzipien des Parlamentarismus?, sowie Michael Eilfort, Geschlossenheit und gute Figur. Ein Versuch über die Steuerung von Fraktionen, beide in: ZParl, 34. Jg. (2003), H. 3, S. 550 - 564 beziehungsweise S. 565 - 582. Gleichwohl ist natürlich nicht auszuschließen, dass einzelne Abgeordnete gegen diesen Grundsatz verstoßen und auf ihrer abweichenden Position beharren beziehungsweise dass eine hinreichend große Minderheit einer Regierungsfraktion ihrer Partei einen Regierungswechsel „aufzwingen“ will. Insofern waren die Vertrauensfragen, die 1982 von Helmut Schmidt und 2001 von Gerhard Schröder gestellt wurden, waren mit einem gewissen Risiko des Scheiterns behaftet.

10 BVerfG, NJW 2005, S. 2669 (S. 2673). 
nicht gehindert, dem Kanzler in einer solchen Situation das Vertrauen auszusprechen, verfassungsrechtlich korrekt, politisch aber wenig wahrscheinlich ist.

Zwar ist die faktische Handlungsfreiheit des einzelnen Abgeordneten vergleichsweise schwach, nicht aber, wie es auf den ersten Blick scheinen mag, die Position der Mehrheitsfraktionen als ganzer. Insofern hat Pieper Recht, wenn er konstatiert, ein Selbstauflösungsrecht würde die Stellung des Bundestages gegenüber dem Kanzler nicht wesentlich stärken. Er bedarf einer solchen Stärkung auch gar nicht, allerdings aus ganz anderen Gründen als den von Pieper genannten: Zwar können sich die Mehrheitsabgeordneten dem öffentlich erklärten Willen des Kanzlers, den Bundestag auflösen zu wollen, kaum widersetzen. Aber auch dieser wird, will er die folgenden Wahlen gewinnen, eine solche Auflösung nicht gegen den Willen der Fraktion durchsetzen. In der Praxis werden sich beide Seiten vielmehr abstimmen, wie dies ja 2005 auch geschehen ist. ${ }^{11}$ Besäße das Parlament - und damit mindestens seine absolute Mehrheit - ein Selbstaufösungsrecht, änderte sich daran nichts: Beide Seiten würden sich wieder miteinander abstimmen, denn ein Auflösungsbeschluss der Parlamentsmehrheit gegen den erklärten Willen der Regierung (de facto also ein Regierungssturz ohne Neuwahl eines Kanzlers ${ }^{12}$ ) wäre nicht nur wenig nachvollziehbar, sondern auch aus wahltaktischen Gründen höchst kontraproduktiv (jedenfalls für die Mehrheitsfraktionen). Insofern könnte auch ein Selbstauflösungsrecht den Bundestag - das heißt also: seine Mehrheit - gegenüber der Regierung nicht wirklich stärken. Man könnte es sogar anders herum formulieren: Entscheidend ist, wer die Idee der Selbstauflösung im konkreten Fall in die Öffentlichkeit bringt. Dies kann natürlich auch durch die Fraktionsführung geschehen, die damit den Kanzler in Zugzwang brächte. Insofern hat das Machtgleichgewicht zwischen Kanzler und Mehrheitsfraktionen zwar eine gewisse „Schlagseite“ zugunsten des Kanzlers von einer "Schwäche“ des Bundestages ihm gegenüber zu reden wäre aber wohl verfehlt. Damit ist zugleich ein Argument gegen ein Selbstauflösungsrecht widerlegt: Es würde zumindest keinen Schaden, das heißt, eine verfassungslogisch nicht intendierte Machtverschiebung zwischen Bundestag und Bundeskanzler mit sich bringen.

Auch die Behauptung, das Selbstauflösungsrecht habe einen „plebiszitären Charakter“, vermag nicht zu überzeugen. Die vorzeitige Beendigung der Wahlperiode führt zu einer (vorgezogenen) Wahl - einem ganz normalen Element des Repräsentativsystems. Auch ihre Vorzeitigkeit kann sie nicht zu einem per se „plebiszitären“ Element machen, jedenfalls nicht, wenn man Plebiszit vor allem als Entscheidung über eine bestimmte Sachfrage versteht. Zwar können, wie 2005 beim Gesetz zu Hartz IV, tatsächlich bestimmte Sachfragen hinter einer vorzeitigen Neuwahl stehen. Entschieden wird doch aber stets - wie in regulären Wahlen auch - über das gesamte Programm der zur Wahl stehenden Parteien. Damit unterscheiden sich vorgezogene Wahlen nicht wesensmäßig von regulären.

11 Allerdings scheint sich in diesem Fall Bundeskanzler Schröder allein mit dem Fraktionsvorsitzenden und Parteichef Franz Müntefering abgesprochen zu haben, ohne dass die Fraktion im Vorfeld darüber informiert worden war. In einem solchen Fall ist das Risiko des „unerwünschten Erfolgs“ der Vertrauensfrage vergleichsweise hoch.

12 So, als de facto destruktives Misstrauensvotum, interpretierte im Übrigen auch der Parlamentarische Rat das von seinem Redaktionsausschuss vorgeschlagene Selbstauflösungsrecht des Bundestages, vgl. Karlheinz Niclauß, Auflösung oder Selbstauflösung? Anmerkungen zur Verfassungsdiskussion nach der Vertrauensfrage des Bundeskanzlers 2005, in: ZParl, 37. Jg. (2006), H. 1, S. 40 -46, S. 44. 
Der fast moralisch zu nennende Impetus, mit dem die Verfassungsväter laut Pieper den Bundestag daran hindern wollten, „sich (...) durch eine vorzeitige Selbstauflösung der Verantwortung zu entziehen"13, mag vor dem Hintergrund der häufigen Auflösungen des Reichstags der Weimarer Republik verständlich sein. ${ }^{14}$ In der heutigen Zeit erscheint eine solche Begründung angesichts der Praxis von Selbstauflösungen in anderen Staaten ${ }^{15}$ und in den deutschen Ländern ${ }^{16}$ nicht realistisch: Keine Parlamentsmehrheit setzt sich willkürlich und ohne jede Not der Gefahr aus, bei anberaumten Neuwahlen ihre Mandatsmehrheit zu verlieren. ${ }^{17}$ Hingegen ist Pieper zuzustimmen, dass kleine Fraktionen majorisiert werden könnten.

Um die Vorteile eines Selbstauflösungsrechts bestimmen zu können, soll zunächst die von Niclauß erneut $^{18}$ ins Spiel gebrachte Variante der vorzeitigen Beendigung der Wahlperiode des Bundestages auf Antrag des Bundeskanzlers ohne formelle Stellung der Vertrauensfrage diskutiert werden. Danach solle ein neuer Art. 68a GG formuliert werden, wonach der Bundeskanzler einen Antrag auf Auflösung des Bundestages stellen könne. Fände dieser die Zustimmung des Bundestages, könne der Bundespräsident das Parlament binnen einundzwanzig Tagen auflösen. Dieses Recht würde wie bisher nach einem erfolgreichen Misstrauensvotum erlöschen. ${ }^{19}$ Eine andere Variante sieht vor, dass der Bundestag zwar aus eigener Initiative einen Auflösungsbeschluss herbeiführen kann, dieser aber einem Zustimmungsvorbehalt des Bundeskanzlers und des Bundespräsidenten unterliegt. ${ }^{20}$

Die Vertreter beider Varianten gehen - anders als Pieper - von der Sinnhaftigkeit, gar der Notwendigkeit einer Verfassungsänderung aus, die eine Auflösung des Bundestages ohne die Stellung der Vertrauensfrage erlaubt. ${ }^{21}$ Das zentrale Argument ist, wie Niclauß sehr pointiert feststellt, das „nichtverstehende Kopfschütteln“ der Bürger bei der Anwendung der vom Bundesverfassungsgericht in einer gewissen Begründungsnot erst als „unecht“, dann als „auflösungsgerichtet“ titulierten Vertrauensfrage. Dabei wissen - jedenfalls die gut informierten - Bürger, dass der Kanzler bei einer solchermaßen „erfolgreich gescheiterten" Vertrauensfrage gar nicht wirklich das Vertrauen seiner Mehrheit verloren haben muss. Spätestens wenn ein solcher Kanzler - wie 2005 geschehen - von eben jenen Partei-

13 Vgl. Stefan Ulrich Pieper, a.a.O., S. 294.

$14 \mathrm{Zu}$ beachten ist allerdings, dass diese Aufösungen nicht auf Beschlüsse des Reichstags, der dazu gar nicht berechtigt war, sondern auf Anordnungen des Reichspräsidenten nach Art. 25 WRV zurückzuführen waren.

15 Vgl. dazu jüngst Werner Patzelt, Vertrauensfrage und Parlamentsaufösung: Parlamentsaufösung im internationalen Vergleich, in: Zeitschrift für Staats- und Europawissenschaften, 4. Jg. (2006), H. 1, S. $121-141$, S. $136 \mathrm{ff}$.

16 Vgl. Sven Leunig, Die Regierungssysteme der deutschen Länder im Vergleich, Opladen 2007, S. 127 - 140. Dies räumt im Übrigen auch Pieper ein, vgl. ders., a.a.O., S. 295.

17 Vgl. in diesem Sinne bereits Hans Peter Bull, Parlamentsauflösung - Zurückverweisung an den Souverän, in: ZRP 9/1972, S. $201-204$.

18 Vgl. bereits Karlheinz Niclauß, Aufösung oder Selbstauflösung?, a.a.O., S. 44.

19 Vgl. ders., Echte und auflösungsorientierte Vertrauensfrage, a.a.O., S. 668. Diese Möglichkeit, die Parlamentsaufösung nur durch Parlamentsbeschluss nach positivem Antrag des Regierungschefs herbeizuführen, bestand unter anderem in Schleswig-Holstein bis 1990. Sie wurde aber nach den Erfahrungen während der Uwe Barschel-Affäre als untauglich abgeschafft.

20 Vgl. Frank Neubauer, Neuwahlen und Grundgesetz, in: DÖV 17/1973, S. 597 - 599.

21 Vgl. ebenda. Letztere solle, so Niclauß, beibehalten, allerdings auf ihre Funktion der Disziplinierung der Mehrheitsabgeordneten beschränkt werden (mit der Möglichkeit der Verbindung mit einer Sachvorlage). 
mitgliedern für die anstehende Neuwahl wiederum auf den Schild des Spitzenkandidaten gehoben wird, oder der Führer der Kanzlerpartei diesem noch während der Abstimmung über die Vertrauensfrage - die ja scheitern soll - des unbedingten Vertrauens seiner Koalitionsfraktionen versichert, setzt dieses Kopfschütteln ein. Die Einzigen, die nach einer solchen Farce das Vertrauen wirklich zu verlieren beginnen, sind die Bürger - und zwar nicht in den Kanzler, sondern, schlimmer, in die Verfassung.

Hinzu kommt, dass die gegenwärtige, wesentlich durch die Interpretation des Bundesverfassungsgerichts geschaffene Rechtslage für die Verfassungsmäßigkeit einer Auflösung des Bundestages nach negativ abgeschlossener Vertrauensfrage voraussetzt, dass es dem Kanzler nach eigener Einschätzung in Zukunft nicht mehr möglich sein wird, eine „auf dem stetigen Vertrauen der Mehrheit des Bundestags“ beruhende Politik umzusetzen. Damit werden die Fälle, in denen entweder der Verlust der Mehrheit zwar denkbar, keineswegs aber wirklich absehbar ist (Helmut Kohl 1983), oder aber gar nicht der Mehrheitsverlust im Parlament, sondern vielmehr der Vertrauensverlust in der Bevölkerung (Gerhard Schröder 2005) hinter dem Wunsch der Parlamentsauflösung steht, nicht abgedeckt, obwohl diese ebenso große „politische Problemlagen“ darstellen können wie der klassische Fall des tatsächlichen Vertrauensverlusts im Parlament. Gleichwohl wurden beide Parlamentsauflösungen - wie im juristischen Schrifttum gelegentlich betont wurde, entgegen den zuvor im selben beziehungsweise vorangehenden Urteil aufgestellten Prinzipien - für verfassungsgemäß erklärt. In seinem Urteil von 2005 wusste sich das BVerfG dann nur noch dadurch zu helfen, dass es zwar bei seiner Definition einer verfassungsgemäßen Auflösungssituation blieb, dessen Vorliegen aber de facto der Einschätzungsprärogative des Bundeskanzlers, hilfsweise des Bundespräsidenten als pouvoir neutre überließ. Wenn diese beiden, und zwischen ihnen auch der Bundestag mit seinem Mehrheitsbeschluss, eine solche Auflösungslage konstatiert haben, müsse das BVerfG wohl von deren Verfassungsmäßigkeit ausgehen. ${ }^{22}$ Diese faktische Selbstentmachtung des Verfassungsgerichts dürfte den bereits angedeuteten Vertrauensverlust der Bürger in die Verfassung und ihre Organe noch verstärken.

Sowohl die von Niclauß favorisierte Lösung einer vorzeitigen Beendigung der Wahlperiode des Bundestages auf Antrag des Kanzlers als auch die Variante eines unabhängigen Selbstauflösungsrechts können diesen drohenden Vertrauensverlust beseitigen.

Für die Aufösung auf Antrag des Bundeskanzlers könnte zunächst sprechen, dass wie bisher zwei Verfassungsorgane neben dem Bundestag eingebunden wären. Das Verfassungsgericht würde dann mangels bestehender materieller Bedingungen für eine Auflösung wohl deutlich an Einfluss verlieren. Dies wäre insofern nicht problematisch, als die Eindeutigkeit der neuen Verfassungslage eine Anrufung des Verfassungsgerichtes eher unwahrscheinlich machen würde.

Allerdings beruht diese positive Bewertung auf einem formalen Verfassungsverständnis, das von drei im Sinne der alten Gewaltenteilungslehre tatsächlich auch politisch voneinander unabhängigen Verfassungsorganen ausgeht. In der Praxis besteht aber in der modernen parlamentarischen Parteiendemokratie bekanntlich ein sehr enges Verhältnis zwischen Regierung(schef) und Parlamentsmehrheit. Schon jetzt ist, wie bereits ausgeführt, eine Auf-

22 Hinzuzufügen bleibt, dass das BVerfG dieselbe Bindungswirkung aus Vertrauensfrage des Bundeskanzlers und eindeutigem Beschluss des Bundestages schon gegenüber dem Bundespräsidenten gegeben sieht, so dass auch dieser kaum noch Möglichkeiten haben dürfte, einem entsprechenden Antrag des Bundeskanzlers auf Auflösung zu widersprechen. 
lösung des Bundestages realistischerweise nur zu erwarten, wenn sich beide Seiten darüber einig sind. ${ }^{23}$ Damit bliebe die Entscheidung über die Verfassungsmäßigkeit beim Bundespräsidenten angesiedelt. Nur: was sollte dieser nach der neuen Verfassungslage überhaupt noch entscheiden? Unter welchen Umständen sollte dieser - mangels dann wohl nicht mehr zu verlangender materieller Bedingungen - einem Auflösungsbeschluss des Bundestages seine Zustimmung verweigern? ${ }^{24}$

Nimmt man tatsächlich zwei politisch voneinander unabhängige Staatsorgane an, so könnte ein Bundeskanzler, der - aus welchen Gründen auch immer - kein Interesse an einer Auflösung des Bundestages hätte, obwohl er faktisch dessen Vertrauen verloren hat, das Parlament daran hindern, seine eigene Wahlperiode vorzeitig zu beenden. Diese Variante mag höchst unwahrscheinlich sein - sie ist aber nicht unwahrscheinlicher als ein zwar zur Regierungsbildung unfähiges, aber partout auflösungsunwilliges, weil ,an seinen Sesseln klebendes" Parlament, das bei Niclauß 3 unter Berufung auf Thomas Dehler als Argument gegen ein Selbstaufösungsrecht herhalten muss. ${ }^{25}$

Insofern spricht mehr für ein unabhängiges Recht des Bundestages, über die vorzeitige Beendigung seiner Wahlperiode selbst entscheiden zu können. In der politischen Praxis wäre der Kanzler dabei in aller Regel eingebunden, ohne das Parlament im Notfall an einer eigenständigen Entscheidung hindern zu können. Die Nicht-Beteiligung des Bundespräsidenten, der auf eine rein notarielle Funktion beschränkt bliebe, ist aus den genannten Gründen wenig problematisch.

In diesem Sinne hat sich auch die Verfassungskommission von 1976 für ein solches Auflösungsrecht ausgesprochen. ${ }^{26}$ Danach sollte der Bundestag in einem diesbezüglich ergänzten Art. 39 Abs. 2 GG das Recht haben, auf Antrag eines Viertels und mit Zustimmung von zwei Dritteln seiner Mitglieder die vorzeitige Beendigung der Wahlperiode zu beschließen. Ein ähnlicher Antrag wurde 1993 von der SPD-Fraktion in der Gemeinsamen Verfassungskommission von Bundestag und Bundesrat gestellt. ${ }^{27}$ Während die Empfehlung der Verfassungskommission 1976, soweit erkennbar, nur auf geringe Resonanz gestoßen ist ${ }^{28}$, konnte sich die SPD 1993 nicht gegen die Stimmen von CDU und FDP durchsetzen. Deren Argumente lauteten im Wesentlichen, ein Selbstauflösungsrecht würde den Zwang, zu politischen Einigungen zu kommen, verringern, und dem Parlament ein verfassungspolitisch zu großes Gewicht zumessen. Überdies hätten die Erfahrungen in anderen europäischen Ländern (Italien, Belgien und Dänemark) gezeigt, dass so auch keine klaren Mehrheitsverhältnisse zu erreichen seien.

23 So auch bereits Hans Peter Bull, a.a.O., S. 203.

24 Einmal ganz abgesehen davon, dass eine solche Verweigerung - also faktisch der Zwang eines auflösungswilligen Parlaments zum Weiterarbeiten - politisch höchst unwahrscheinlich sein dürfte.

25 Vgl. Karlheinz Niclauß, Auflösung oder Selbstauflösung?, a.a.O., S. 46.

26 Vgl. Enquete-Kommission Verfassungsreform des deutschen Bundestages, Schlussbericht, BT-Drs. 7/5924, S. $39 \mathrm{ff}$.

27 Vgl. Bericht der Gemeinsamen Verfassungskommission, BT-Drs. 12/6000 vom 5. November 1993, S. 87. Abweichend wurde hier von einem Antragsquorum von einem Drittel ausgegangen.

28 Zwar hatte sich auch die CDU/CSU-Fraktion in ihrem Entschließungsantrag zum Schlussbericht der Verfassungskommission ausdrücklich empfehlend für die Aufnahme eines Selbstauflösungsrechts geäußert, vgl. BT-Drs. 8/1517. Ebenso nahm im Bundestag als Einziger ausdrücklich der Abgeordnete Friedrich Wendig (FDP) zu dieser Empfehlung positiv Stellung. Aus Bericht und Debatte folgte aber keine Beschlussvorlage einer der Bundestagsfraktionen, vgl. BT-PIProt. 73/8, S. $5776(\mathrm{C})$. 
$\mathrm{Zu}$ den beiden ersten Argumenten wurde bereits oben Stellung genommen: Sie verkennen erstens, dass sich nicht einigungsbereite Fraktionen letztlich auch durch Verfassungsbestimmungen nicht zur Kreation beziehungsweise Unterstützung einer stabilen Regierung zwingen lassen. Zweitens geht die Vorstellung von politisch „unabhängigen“ Verfassungsinstanzen von einem rein formalen Verfassungsverständnis aus, das die enge Verknüpfung von Bundestagsmehrheit und „ihrem“ Kanzler außer Acht lässt. Drittens kann der Verweis auf die Situation in den genannten Ländern am wenigsten überzeugen: Die häufigen Regierungswechsel in Italien bis zu Beginn der 1990er Jahre etwa waren keineswegs auf vorzeitige Beendigungen der Wahlperioden zurückzuführen, sondern auf eine davon völlig unabhängige Instabilität des italienischen Parteiensystems.

In der Frage der notwendigen Mehrheit, die einer Selbstauflösung zustimmen muss, spricht gerade die auch von Pieper angeführte ansonsten drohende Majorisierung kleinerer Parteien für ein im Vergleich zu sonstigen Parlamentsbeschlüssen erhöhtes Quorum (zwei Drittel). Bemerkenswert ist, dass in fast allen Bundesländern, in denen es sowohl ein Selbstaufösungsrecht mit qualifizierter Mehrheit als auch die Möglichkeit der mit einfacher Mehrheit abzulehnenden Vertrauensfrage gibt, letztere bislang nie genutzt wurde, um eine Parlamentsauflösung durch die Mehrheitsfraktionen gegen den Willen der größeren Oppositionsfraktion durchzusetzen. ${ }^{29}$ Hier hat sich also die Selbstauflösung als ein eher konsensual genutztes Mittel gezeigt. In jedem Fall würde es den bisher notwendigen, für die Bürger kaum nachvollziehbaren Umweg über die Vertrauensfrage ersparen, wenn ohnehin die große Mehrheit aller Parlamentsparteien der Meinung ist, Neuwahlen anberaumen zu müssen.

29 Vgl. Sven Leunig, a.a.O., S. 127 - 140.

\title{
Ein Blick über die Grenzen hilft manchmal weiter. Eine Replik auf Gerd Strohmeier in Heft 3/2007 der ZParl ${ }^{*}$
}

\author{
Stefan Köppl
}

Gerd Strohmeiers Vorschlag zu einer Reform des deutschen Wahlrechts hat bereits massive Kritik geerntet. Frank Decker zeigte in seiner Replik, dass Strohmeiers Problemdiagnose unvollständig beziehungsweise unzutreffend, seine Idealvorstellungen fragwürdig und seine Reformvorschläge systemwidrig sowie unrealistisch seien. ${ }^{1}$ Zudem rechnete Harald Schoen nach, dass die Vorschläge kaum dazu geeignet sind, ihre Ziele zu erreichen. ${ }^{2}$ Dem soll an

* Gerd Strohmeier, Ein Plädoyer für die ,gemäßigte Mehrheitswahl“: optimale Lösung für Deutschland, Vorbild für Österreich und andere Demokratien, in: ZParl, 38. Jg. (2007), H. 3, S. 578 $-590$.

1 Frank Decker, Konsens- oder mehrheitsdemokratischer Wandel des Parlamentarismus? Eine Replik auf Gerd Strohmeier in Heft 3/2007 der ZParl, in: ZParl, 38. Jg. (2007), H. 4, S. 857 - 861.

2 Harald Schoen, Eine optimale Lösung? Eine Replik auf Gerd Strohmeier in Heft 3/2007 der ZParl, in: ZParl, 38. Jg. (2007), H. 4, S. $862-865$. 